\title{
Cumulative proactive interference in animal memory
}

\author{
WENDY V. EDHOUSE \\ Otago University, Dunedin, New Zealand \\ and Victoria University of Wellington, Wellington, New Zealand \\ and \\ K. GEOFFREY WHITE \\ Otago University, Dunedin, New Zealand
}

\begin{abstract}
Events occurring on the prior trial in delayed matching-to-sample tasks can proactively interfere with accurate matching on the current trial. The present study investigated the accumulation of proactive interference in delayed matching-to-sample at the local level of two consecutive trials, as well as in terms of a general performance decrement accumulating over the session. Higher-order analyses, in terms of the parameters of negative exponential functions fitted to the data, showed that the magnitude of the local proactive-interference effect resulting from intertrial disagreement of stimuli decreased over the session. Furthermore, there was no evidence for the general performance decrement over the session, which is frequently attributed to proactive interference. The attenuation of the local proactive-interference effect was accounted for in terms of changes in the relative probabilities of agreeing and nonagreeing trials.
\end{abstract}

In a previous study, we demonstrated a specific or local proactive-interference effect in which delayed matching-to-sample (DMTS) performance on one trial was interfered with by conflicting events on the immediately preceding trial (Edhouse \& White, 1988). This local proactive-interference effect was manifest as a higher rate of forgetting when the sample stimuli differed between consecutive trials than when the sample stimuli remained the same between consecutive trials.

We also demonstrated that the local proactive effect was independent of the temporal separation or intertrial interval (ITI) between successive trials. Decreasing the ITI, however, had an overall effect of impairing performance-a general proactive-interference effect. Therefore, the performance decrement resulting from shortening the ITI duration cannot be accounted for by local proactive-interference effects, consistent with the results of two previous studies (Roberts, 1980; Roitblat \& Scopatz, 1983). We further demonstrated the independence of local and general proactive-interference effects by showing that interpolating a houselight into the ITI eliminated the general effect while leaving the local effect intact (Edhouse \& White, 1988). Although our previous study examined proactive interference only for pairs

This research was supported by a University Grants Committee Postgraduate Scholarship awarded to W. V. Edhouse, and by grants from the University Grants Committee to K. G. White. These data were reported at the Experimental Analysis of Behavior Symposium of the New Zealand Psychological Society Conference held in Wellington, 1987. Reprint requests should be sent to Wendy V. Edhouse, Department of Psychology, Victoria University of Wellington, P.O. Box 600 , Wellington, New Zealand, or to K. G. White, Department of Psychology, Otago University, Dunedin, New Zealand. of consecutive trials, trials prior to Trial $n-1$ may also influence performance on Trial $n$; that is, proactive interference that accumulates over a session may add to the effect of conflicting events on immediately preceding trials.

In a study of human short-term memory, Keppel and Underwood (1962, Experiment 3) found that the performance decrement assumed to result from proactive interference persisted beyond the fifth item. With short delay intervals, performance declined over the first four items and then increased at the fifth and sixth items, suggesting a practice effect. With a long (18-sec) delay, performance progressively declined across items one to six; that is, proactive interference accumulated over the six items at the long delay interval, whereas no such effect was present at the short delay. With a short delay, Loess (1964) also found an increase in proactive interference over the first few trials.

Apparently, few researchers in the animal memory field have directly investigated changes in performance over a session. Previous researchers using variations of the DMTS procedure and pigeons or monkeys as subjects have commented that it is unlikely that trials prior to Trial $n-1$ would have any effect on Trial $n$ performance (Hogan, Edwards, \& Zentall, 1981; Reynolds \& Medin, 1981). This assumption has been based on temporal factors; for example, Hogan et al. suggested,

The present study found that the effects of Trial $n-1$ on performance on Trial $n$ were virtually absent following a 10-sec ITI (especially in phase 1), and, since the end of Trial $n-2$ was separated from the start of Trial $n$ by from 10 to $25 \mathrm{sec}$, it is unlikely that trials prior to Trial $n-1$ had any effect on Trial $n$ performance. (1981, p. 399) 
Despite a lack of direct investigation, several researchers have suggested that a performance decrement may occur in DMTS performance by pigeons due to accumulated effects of prior events across the session. Roberts (1980, Experiment 4) found that pigeons' DMTS performance was superior when trials containing stimuli from a different dimension were embedded in a session than when no such embedded trials occurred. Roberts suggested that the massed-trial effect (performance decrement found with short ITIs) may have resulted from the "massed presentation of the same information on successive trials" (Roberts, 1980, p. 234).

Grant (1976) found no evidence for intertrial proactive interference either from Trial $n-1$ or from Trial $n-2$, and suggested that significant interference may be generated by a more general source of proactive interference. He suggested that interference could build up across trials within a session so that a "collection of prior trials" (Grant, 1976, p. 587), rather than a particular trial, produced interference.

Wright, Urcuioli, and Sands (1986) developed an argument, largely on the basis of their findings with monkeys and pigeons in the serial-probe recognition task, that a performance decrement results from an accumulation of proactive interference when trial events are repeated in a session. These researchers found low performance levels when sample set size was small (resulting in the necessary repetition of the small number of stimuli) compared with performance levels when trial-unique stimuli were used (i.e., no item in the sample set was shown more than once). Wright et al. suggested that a general, pervasive proactive effect "might develop if [proactive interference] accumulates across successive memory trials" (1986, p. 108).

Roberts and Dale (1981) found that proactive interference in rats on a spatial memory task increased over the first three trials in a session, with the performance decrement being greater at the longer delay interval. Interestingly, this finding parallels that for the early human shortterm memory studies. Pontecorvo (1983) also used rats to investigate the buildup of proactive interference in continuous matching-to-sample. He found that accuracy increased as a function of the number of trials since the last "interfering" (nonmatching) trial; however, delay did not interact with the number of trials since the last nonmatch trial, suggesting that rate of forgetting was unaffected by this form of proactive interference.

Wilkie (1986) used a spatial memory task in possibly the only investigation into the buildup of proactive interference in pigeons over the entire session. Wilkie measured performance over blocks of trials within a session. He found no evidence that performance changed over the session, but he did not manipulate delay interval. It is impossible, therefore, to conclude whether there may have been a change in local proactive interference which is manifest as a difference in rate of forgetting (Edhouse \& White, 1988).

In a second experiment, Wilkie (1986) manipulated the distractor set so that the incorrect choice on Trial $n$ either was the correct choice on Trial $n-1$ or had not been present on Trial $n-1$. However, Wilkie did not record performance across session blocks. It is impossible, therefore, to tell whether the local proactive interference that did occur, changed over the session.

In the current study, DMTS performance of pigeons was examined over four successive 16-trial blocks of the session, in a procedure that also permitted the analysis of the local proactive-interference effect. We investigated the possibility that local proactive interference gradually accumulates over a session (see Roberts, 1980).

\section{METHOD}

\section{Design}

The present experiment employed a within-subjects factorial design, with the factors consisting of four levels of delay interval $(0.5$, $5,10$, and $20 \mathrm{sec})$, four successive quarters of a session $(1,2,3$, and 4), and four levels of sequence (relation of correct choice stimulus on Trial $n$ to correct choice stimulus on Trial $n-1$ : same wavelength and same left or right position, same wavelength and different position, different wavelength and same position, and different wavelength and different position).

\section{Subjects}

Five adult homing pigeons with prior experience in delayed matching-to-sample procedures (White, 1985; White \& BunnellMcKenzie, 1985; White \& McKenzie, 1982) were maintained at $80 \% \pm 15 \mathrm{~g}$ of their free-feeding weights. Water and grit were always available in the living cages.

\section{Apparatus}

A sound-attenuating experimental chamber (Lehigh Valley), with internal dimensions of $31 \times 34 \times 33 \mathrm{~cm}$, contained a three-key intelligence panel. The interior was painted matte black. A houselight on a side wall was unlit throughout the experiment. The hopper opening was beneath the center key. An exhaust fan provided general masking noise. The three translucent response keys were $2.5 \mathrm{~cm}$ in diameter; $10 \mathrm{~cm}$ apart, center to center; and $23 \mathrm{~cm}$ above the grid floor. Closure of microswitches mounted behind the keys required pressures of $.15 \mathrm{~N}$ for the center key and $.20 \mathrm{~N}$ for the side keys.

In-line projectors (IEE with 24-V 1820 lamps) were mounted behind the keys. Sample and comparison stimuli presented on the center and side keys were $0.8-\mathrm{cm}$ disks of color produced by Kodak Wratten filters 74 and 73 with peak transmission at wavelengths of $538 \mathrm{~nm}$ and $576 \mathrm{~nm}$, respectively. Kodak neutral density filters were added to the Wratten filters to equate brightness of both color stimuli on all three keys at $0.2 \mathrm{~cd} / \mathrm{m}^{2}$ as measured by a Pentax Spotmeter.

Delay intervals were timed by a solid-state time base and decade counters, and trial sequences and other events were controlled and recorded by a tape reader, solid state and relay circuitry, and digital counters, all located in an adjacent room.

\section{Procedure}

Owing to their prior training, the subjects showed a high level of matching accuracy at the beginning of the experiment, and thus did not require pretraining. Each trial of the current procedure began with presentation of the center key illuminated with a yellow (576-nm wavelength) or a green (538-nm wavelength) sample stimulus. The fifth peck darkened the key and initiated a delay interval that lasted for $0.5,5,10$, or $20 \mathrm{sec}$. During the delay interval, the chamber was dark and responses were ineffective. The delay interval terminated with the onset of comparison stimuli on the side keys, one green (538-nm wavelength) and the other yellow (576-nm 
wavelength). Correct (matching) responses were the choice of the yellow comparison stimulus following the yellow sample and the choice of the green comparison stimulus following the green sample. A single correct response darkened both keys, produced 2-sec access to grain, and initiated an intertrial interval that lasted for $10 \mathrm{sec}$.

Each daily session comprised 65 trials, 64 of which were used in data analysis. The first trial each day was a "dummy" trial. Orders of sample-stimulus wavelength and position of correct comparison stimulus (left or right) were randomized within each session. Each wavelength value was used equally often as a sample stimulus and as the correct and incorrect comparison stimulus. Furthermore, the right-left position of the correct comparison stimulus was balanced across types of trials, and each type of trial was tested an equal number of times with a $0.5-, 5-, 10-$, and $20-\mathrm{sec}$ delay.

Each session included four types of intertrial sequence. The relation of the sample-stimulus wavelength, and hence the correct comparison-stimulus wavelength, and the left or right position of the correct comparison stimulus on Trial $n$ to the sample-stimulus wavelength and the position of the correct comparison stimulus on Trial $n-1$ can be organized into four different two-trial combinations, henceforth referred to in this paper as Sequences I to IV. Sequence I refers to two-trial combinations in which both the sample-stimulus wavelengths and the positions of the correctcomparison stimulus are the same on two consecutive trials. Sequence II refers to two-trial combinations in which sample-stimulus wavelengths on Trials $n$ and $n-1$ are identical but in which the position of the correct comparison stimulus on Trial $n$ differs from that on Trial $n-1$. Sequence III refers to two-trial combinations in which the sample-stimulus wavelength on Trial $n$ differs from that on Trial $n-1$ (hence the correct comparison-stimulus wavelength of Trial $n-1$ is the incorrect comparison-stimulus wavelength of Trial $n$ ) but in which the position of the correct comparison stimulus is identical on Trials $n$ and $n-1$. Sequence IV refers to two-trial combinations in which both sample-stimulus wavelength and the position of the correct comparison stimulus differ between Trials $n$ and $n-1$.

Combining the two possible stimulus wavelengths and the two possible positions of correct comparison stimulus on Trial $n$ with the two possible stimulus wavelengths and the two possible positions of correct comparison stimulus on Trial $n-1$ provided four ways of representing each sequence. For example, Sequence I was represented by two consecutive trials in which sample stimuli were yellow and the yellow comparison stimuli were on the left, or sample stimuli were yellow and the yellow comparison stimuli on the right, or sample stimuli were green and the green comparison stimuli were on the left, or sample stimuli were green and the green comparison stimuli were on the right. Similarly, other sequences were represented by four combinations of consecutive trials. The four ways of combining two trials to produce each sequence, combined with the four types of sequences and the four delay intervals, provided 64 different combinations of these factors. These 64 combinations were presented over a session and were repeated four times in each 4-session block. The specific combinations of consecutive trials occurred in the 1st, 2nd, 3rd, and 4th quarters of each of the four sessions in a block. The order of the specific combinations and the different quarters of each session were balanced over blocks of four sessions and over subjects. The experiment was conducted for a total of 24 sessions.

\section{RESULTS}

Performance was first measured in terms of the proportion of correct responses, that is, the number of correct responses divided by the total number of responses for a given trial type. The data were collapsed over Sequences I and II (same sample stimuli on consecutive trials) and over Sequences III and IV (different sample stimuli on consecutive trials), since in two previous experiments (Edhouse \& White, 1988) positional information was not a significant factor in proactive interference. Proportions correct were calculated for each bird from the $\mathbf{4 8}$ trials conducted over the 24 sessions with each of the 32 combinations of delay interval, session quarter, and sequence. The total of 160 observations (each based on 48 trials) over the 5 birds was submitted to a $4 \times 4 \times 2$ analysis of variance for repeated measures on all three factors.

There were significant main effects of delay $[F(3,12)$ $=10.74, p<.001]$ and sequence $[F(1,4)=34.04, p<$ $.01]$. The main effect of session quarter was not significant $[F(3,12)=2.50, p>.05]$. There were no significant interactions.

Figure 1 shows performance on each session quarter as a function of delay interval. Accuracy was lowest in the first quarter of the session; mean proportions correct for successive quarters were $0.658,0.750,0.754$, and 0.734 . After the first quarter, there is no evidence that overall performance changed as the session progressed. To investigate the possibility of a rapid buildup in general proactive interference, performance on the first few trials was examined. Collapsed over delays, percent correct on the first trial of each session averaged 0.47 ; over the next five trials, mean percent correct was 0.60 , compared with 0.66 for the first quarter of the session. This apparent "warm-up" in performance (Spear, 1978; Thomas, 1981) is consistent with the increase in overall accuracy from the first quarter to the later quarters of the session, but not with a buildup in general proactive interference.

Figure 2 shows the effects of delay and sequence over session quarter. Performance declined with increasing delay interval. The effect of sequence was manifest as higher performance, particularly at the longer delays, for Sequences I and II than for Sequences III and IV. The sequence effect demonstrates the specific localized form of

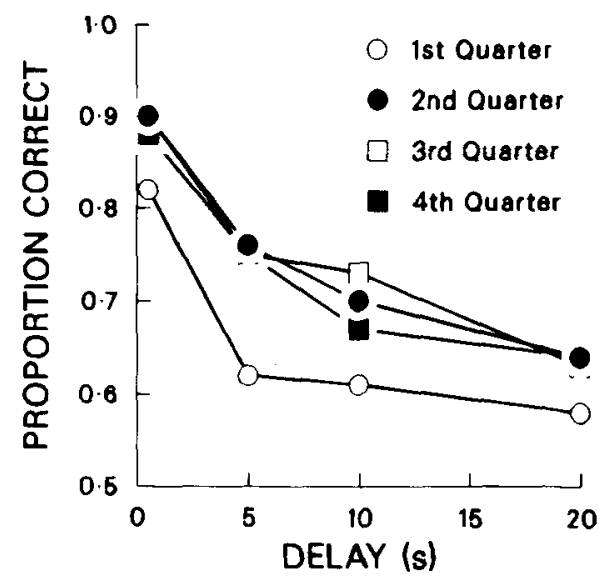

Figure 1. Mean proportion correct as a function of delay (in seconds) for each quarter of the session. 

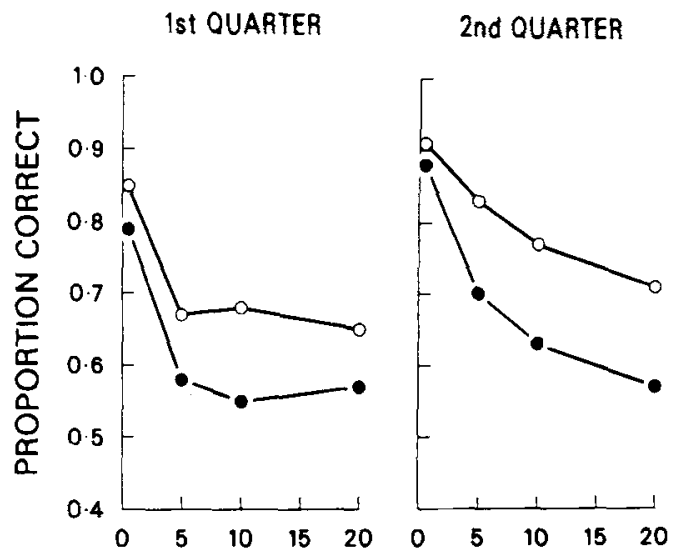

3rd QUARTER

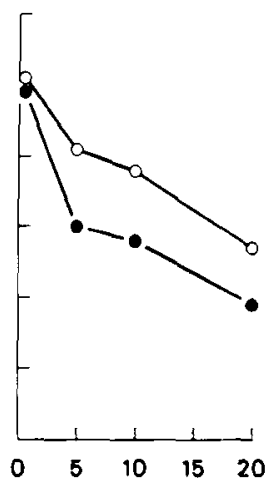

4th OUARTER

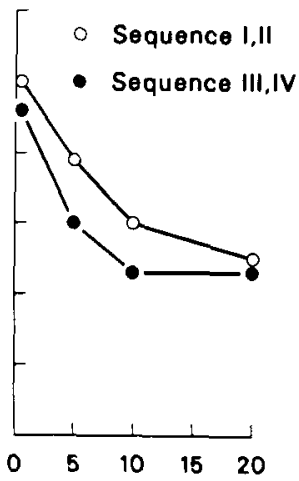

DELAY (s)

Figure 2. Mean proportion correct as a function of sequence and delay (in seconds) for each quarter of the session: Sequence I (same wavelength, same position), Sequence II (same wavelength, different position), Sequence III (different wavelength, same position), and Sequence IV (different wavelength, different position).

proactive interference, described by Edhouse and White (1988). Accuracy was lower when the Trial $n-1$ sample differed from the Trial $n$ sample (i.e., Sequences III and IV) than when the samples on Trials $n$ and $n-1$ were the same (Sequences I and II). Figure 2 indicates that the sequence effect was attenuated as the session progressed, although the quarter $\times$ delay $\times$ sequence interaction failed to reach significance $[F(9,36)=0.43, p>.05]$; that is, the advantage of Sequences I and II over Sequences III and IV is most pronounced early in the session and subsequently declines.

A possible buildup in local proactive interference in the first few trials was examined, although the different delays and sequence types were not perfectly balanced over the first few trials. Over Trials 2 to 6 , mean percent correct averaged over all delays was not different for Sequences I and II (0.58) than for Sequences III and IV (0.64). Compared with the large local proactiveinterference effect in the first quarter of the session, the absence of a local effect in Trials 2 to 6 indicates that local proactive interference must build up rapidly in the first quarter of each session.

To summarize, the present results provide no evidence that an increase in the local proactive-interference effect across the session is a source of cumulative proactive interference. To the contrary, the local proactiveinterference effect appears to attenuate as the session progresses.

To examine the changes in performance across a session in more detail, we used an analysis that summarizes the forgetting functions in terms of two higher-order measures: discriminability of the sample stimulus at zero delay and rate of performance decrement over time since sample presentation. For this analysis, performance was assessed in terms of discriminability of the sample stimuli at the different delay intervals (White, 1985; White \& McKenzie, 1982). The discriminability measure is called $\log d$ and was derived from Davison and Tustin's (1978) analysis of signal detection performance. $\log d$ is analogous to $d^{\prime}$ of signal detection theory, and is not subject to bias, whereas the proportion correct measure used above is (McCarthy \& White, 1987). $\log d$ is equivalent to the detection measure proposed by Luce (1963).

$\log d$ is computed from the frequencies of choice responses to comparison stimuli following samples $S 1$ and S2. Correct response frequencies following S1 and S2, respectively, are P11 and P22. Errors following S1 and $S 2$, respectively, are $\mathrm{P} 12$ and $\mathrm{P} 21$. The index of discriminability at a given delay interval is

$$
\log d=1 / 2[\log (\mathrm{P} 11 / \mathrm{P} 12)-\log (\mathrm{P} 21 / \mathrm{P} 22)] .
$$

White (1985) and White and McKenzie (1982) suggested that the effect of the delay in delayed matching-to-sample procedures is to attenuate discriminability as a negative exponential function of delay duration $(t)$; that is,

$$
\log d=\log d_{0} \cdot \exp (-\mathrm{bt}),
$$

where $\log d_{0}$ is discriminability at zero delay and $b$ is the rate of decrement in discriminability, with dimensions of $t^{-1}$.

The advantage of quantifying delayed matching in terms of the negative exponential function is that two higherorder measures of performance are afforded: discriminability of the sample stimuli in the absence of a memory requirement $\left(\log d_{0}\right)$ and the rate of forgetting $(b)$ (i.e., the rate at which matching accuracy decreases with increasing delay-interval duration; White, 1985). Thus, a "forgetting function" that relates discriminability to delay-interval duration is summarized by the parameters $\log d_{0}$ and $b$.

Discriminability measures at each delay interval, $\log d$, were derived from response frequencies summed over birds according to Equation 1. The negative exponential function given by Equation 2 was fitted to data for each 
set of delay intervals by nonlinear least-squares regression. The changes of interest are in the parameter values, $\log d_{0}$ and $b$.

Figure 3 shows exponential functions fitted to the $\log d$ measures with values for the $\log d_{0}$ and $b$ parameters and proportion of variance accounted for (VAC) for each function. The forgetting functions in Figure 3 describe performance for the four successive quarters of a session. Initial discriminability is lower in the first quarter than in the other three session quarters, in which performance is essentially the same. There is no evidence to suggest that a gradual accumulation of proactive interference results in a performance deficit that increases over a session.

Figure 3 also shows the effects of quarter, delay, and sequence. The size of the local proactive-interference effect, measured by the difference in forgetting rates for Sequences I and II compared with those for Sequences III and IV, decreases over the session. It can be seen that there is no change in rate of forgetting over session quarters for Sequences I and II, whereas for Sequences III and IV there is a systematic reduction in forgetting rate as each session progresses. These conclusions are confirmed by an analysis of fits to Equation 2 of individual data. There was a significant effect of sequence on the $b$ parameter $[F(1,4)=9.53, p<.05]$ and an interaction

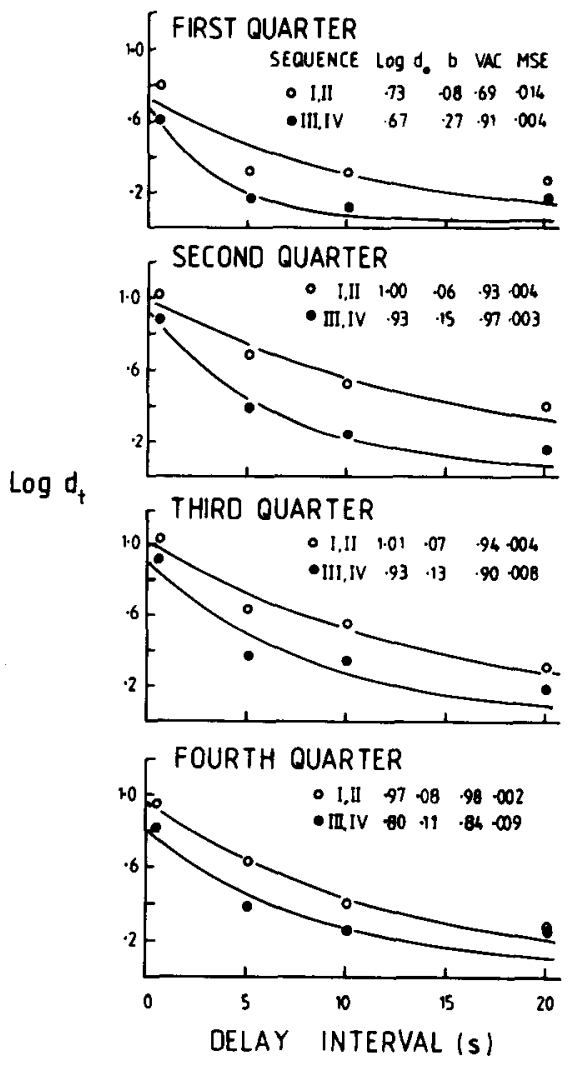

Figure 3. Discriminability as a function of delay and sequence for 1st, 2nd, 3rd, and 4th quarters of the session. Parameter values for best fitting exponential functions are shown. See text for additional information.
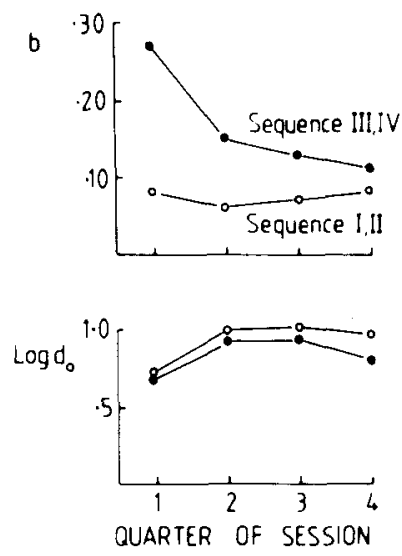

Figure 4. Parameters of the negative exponential forgetting functions as a function of sequence and session quarter.

between type of sequence and session quarter $[F(3,12)$ $=3.54, p<.05$ ]

It is concluded, therefore, that the more rapid rate of forgetting that occurs on conflicting sequences is attenuated as the session progresses. The cumulative effect of prior-trial events results in a reduction in the local proactive-interference effect.

Figure 4 shows the changes in parameters of the negative exponential forgetting functions over the four successive quarters of a session. Initial discriminability $\left(\log d_{0}\right)$ is lower on the first quarter, and then remains essentially the same for the remainder of the session both for Sequences I and II and for Sequences III and IV $[F(3,12)=3.92, p<.05]$. On the other hand, the converging functions in the upper panel of Figure 4 show that the type of sequence has a marked effect on rate of forgetting $(b)$; the steeper rate of forgetting found for Sequences III and IV was most evident early in the session and was subsequently attenuated. There is minimal change in $b$ for Sequences I and II, although after the first quarter there is a trend for $b$ to increase.

\section{DISCUSSION}

These data provide evidence of an attenuation, rather than an accumulation, over a session in the local form of proactive interference identified in our previous studies. The present results clearly provide no support for the hypothesis that a pervasive proactive-interference effect builds up over the session as the number of prior items (or their repetition) increases (Roberts, 1980). Rather, they are more in keeping with a general warm-up in performance over the first trials of each session, combined with a reduction in the local proactive interference resulting from intertrial disagreement. Over the first five trials of each session, there was no evidence for local proactive interference in that performance was similar for Sequences I and II and for Sequences III and IV. A full analysis of the first few trials in terms of exponential function parameters was not possible, however. Furthermore, the overall low level of performance over the first few trials 
may have masked the local proactive-interference effect Nevertheless, the data are consistent with the conclusion that the local proactive-interference effect builds up rapidly from the first few trials in the session, and then declines as the session progresses beyond the first quarter.

A temporal discrimination hypothesis (D'Amato, 1973) would not predict a change in the specific or local proactive-interference effects after the first few trials; as long as the temporal parameters remain constant across the session, the degree of temporal discriminability between successive trials, or across the session, would not be expected to change. Similarly, classical interference theory and a trace-decay model of the type proposed by Roberts and Grant (1976) to describe pigeon DMTS performance can also both easily account for the failure to find an increase over the session in the performance decrement assumed to result from proactive interference. In the former case, an accumulation of proactive interference is assumed to result from an increase in the number of possibly interfering items that may spontaneously recover with time. Hence, in the current study, which employs two-alternative DMTS (sample set size $=2$ ), the number of interfering items does not increase after the first few trials. In the case of trace-decay theory, traces of prior items further back than a few trials would have decayed and no longer be "interfering."

However, these models also predict that the specific interference from the prior-trial stimuli results in a corresponding constant performance impairment across a session after the first few trials, and cannot so easily accommodate the finding of an attenuation in the proactive-interference effect localized at the level of two successive trials.

In the trace-decay model (Roberts \& Grant, 1976), the assumptions of trace independence and trace competition do not allow the model to predict a change in performance with increasing numbers of prior trials. As long as the temporal gap between trials remains constant throughout the session, this should control the retention loss so that prior items will decay and their strength will decrease at a constant rate over a session. Therefore, the relationship between Sequences I and II and Sequences III and IV would not be expected to change after the first few trials. Even if the model were modified to allow traces to "interact," so that "traces of prior items will accelerate the rate at which the trace of the current TBRI [to-beremembered-item] is degraded"' (Bennett, 1975, p. 126), this would allow a decrease in retention with increasing numbers of prior trials but would still not account for an improvement in performance on conflicting sequences across the session, as found in the current study.

Within the classical interference framework, the increase in forgetting as delay interval increases in duration is assumed to result from interference produced by the spontaneous recovery of unlearned prior items. As the number of prior items increases, so does the number of possibly interfering items, resulting in a performance decrement. Classical interference theory would therefore not predict proactive interference to necessarily be maximal after the first few trials. In the current situation, twoalternative DMTS, the number of possibly interfering items is never greater than one, although the number of times this item has been previously seen increases as the session progresses. Hence, this model could account for either no change or an increase in the local proactiveinterference effect as the session progresses, but makes no provision for an attenuation of the effect.

The attenuation of the localized proactive-interference effect over the latter half of the session can be accounted for within an interference framework by a retrieval model of proactive interference in DMTS. In this model, memory "resets" at trial end (Honig, 1978), but interfering information from prior-trial events is retrieved to affect the Trial $n$ choice. A buildup of interference occurs early in the session, as the result of the incorrect choice stimulus serving as a retrieval cue for the previously seen sample stimulus (Wright, Urcuioli, \& Sands, 1986). Such a buildup of proactive interference at the beginning of the session should be very rapid, however, and subsequent gradual attenuation is expected in terms of the current result. Future research, employing a design that attempts to eliminate the warm-up decrement observed in the current study, could provide a direct test of this assumption.

An attenuation of the local proactive-interference effect can be accounted for in terms of relative probabilities of agreeing and nonagreeing prior trials; that is, since the local proactive-interference effect is the result of Trial $n-1$ events differing from those of Trial $n$, and since a long $(20-\mathrm{sec})$ ITI has been found to not reduce these local effects (Edhouse \& White, 1988), earlier trials $(n-2, n-3$, and so forth) may also influence performance. However, as trials progress, Trial $n$ is preceded by an increasingly larger number of both same and different trials. Consequently, as the trials go further back, both Sequence I and II and Sequence III and IV trials are preceded by equiprobable agreeing or nonagreeing trials. If these trials influence Trial $n$ performance, their effect will get more similar with increasing prior trials. Hence, an attenuation in the sequence effect should result as the session progresses.

\section{REFERENCES}

Bennett, R. W. (1975). Proactive interference in short-term memory: Fundamental forgetting processes. Journal of Verbal Learning \& Verbal Behavior, 14, 123-144.

D'Aмato, M. R. (1973). Delayed matching and short-term memory in monkeys. In G. H. Bower (Ed.), The psychology of learning and motivation: Advances in research and theory (Vol. 7, pp. 227-269). New York: Academic Press.

Davison, M. C., \& Tustin, R. D. (1978). The relation between the generalized matching law and signal-detection theory. Journal of the Experimental Analysis of Behavior, 29, 331-336.

Edhouse, W. V., \& WHTE, K. G. (1988). Sources of proactive interference in animal memory. Journal of Experimental Psychology: Animal Behavior Processes, 14, 56-70.

Grant, D. S. (1976). Effect of sample presentation time on long-delay matching in the pigeon. Learning \& Motivation, 7, 580-590. 
Hogan, D. E., Edwards, C. A., \& Zentall, T. R. (1981). Delayed matching in the pigeon: Interference produced by the prior delayed matching trial. Animal Learning \& Behavior, 9, 395-400.

HoNIG, D. E. (1978). Studies of working memory in the pigeon. In S. H. Hulse, H. Fowler, \& W. K. Honig (Eds.), Cognitive processes in animal behavior (pp. 211-248). Hillsdale, NJ: Erlbaum.

KePPEL, G., \& UNDERWOOD, B. J. (1962). Proactive inhibition in shortterm retention of single items. Journal of Verbal Learning \& Verbal Behavior, 1, 153-161.

LoEss, H. (1964). Proactive inhibition in short-term memory. Journal of Verbal Learning \& Verbal Behavior, 3, 362-368.

LuCE, R. D. (1963). Detection and recognition. In R. D. Luce, R. R. Bush, \& E. Galanter (Eds.), Handbook of mathematical psychology (Vol. 1, pp. 103-189). New York: Wiley.

MCCARThY, D., WhITE, K. G. (1987). Behavioral models of delayed detection and their application to the study of memory. In M. L. Commons, J. A. Nevin, \& H. C. Rachlin (Eds.), Quantitative analysis of behavior. Vol. 5: The effect of intervening events on reinforcement value (pp. 29-54). Cambridge, MA: Ballinger.

PONTECORVO, M. J. (1983). Effects of proactive interference on rats' continuous nonmatching-to-sample performance. Animal Learning \& Behavior, 11, 356-366.

Reynolds, T. J., \& Medin, D. L. (1981). Stimulus interaction and between-trials proactive interference in monkeys. Journal of $E x$ perimental Psychology: Animal Behavior Processes, 7, 334-347.

ROBERTS, W. A. (1980). Distribution of trials and intertrial retention in delayed matching to sample with pigeons. Journal of Experimental Psychology: Animal Behavior Processes, 6, 217-237.

RoberTs, W. A., DALE, R. H. I. (1981). Remembrance of places lasts: Proactive inhibition and patterns of choice in rat spatial memory. Learning \& Motivation, 12, 261-281.
ROBERTs, W. A., Grant, D. S. (1976). Studies of short-term memory in the pigeon using the delayed matching-to-sample procedure. In D. L. Medin, W. A. Roberts, \& R. T. Davis (Eds.), Processes of animal memory (pp. 79-112). Hillsdale, NJ: Erlbaum.

RoITBLAT, H. L., SCOPATZ, R. A. (1983). Sequential effects in pigeon delayed matching-to-sample performance. Journal of Experimental Psychology: Animal Behavior Processes, 9, 202-221.

SPEAR, N. E. (1978). Processing of memories: Forgetting and retention. Hillsdale, NJ: Erlbaum.

Thомаs, D. (1981). Studies of long-term memory in the pigeon. In N. E. Spear \& R. R. Miller (Eds.), Information processing in animals: Memory mechanisms (pp. 257-290). Hillsdale, NJ: Erlbaum.

WHITE, K. G. (1985). Characteristics of forgetting functions in delayed matching to sample. Journal of the Experimental Analysis of Behavior, 44, 15-34.

White, K. G., \& Bunnell-McKenzie, J. (1985). Potentiation of delayed matching with variable delays. Animal Leaming \& Behavior, 13, 397-402.

WHITE, K. G., \& MCKENZIE, J. (1982). Delayed stimulus control: Recall for single and relational stimuli. Journal of the Experimental Analysis of Behavior, 38, 305-312.

WiLKIE, D. M. (1986). Pigeons' spatial memory: V. Proactive interference in the delayed matching of key location paradigm occurs only under restricted conditions. Animal Leaming \& Behavior, 14, 257-266.

Wright, A. A., Urcuion, Р. J., SAndS, S. F. (1986). Proactive interference in animal memory. In D. F. Kendrick, M. E. Rilling, \& M. R. Denny (Eds.), Theories of animal memory (pp. 101-125), Hillsdale, NJ: Erlbaum.

(Manuscript received September 10, 1987; revision accepted for publication April 6, 1988.) 